\title{
FUNDAMENTOS DEL PARADIGMA CUALITATIVO EN LA INVESTIGACION EDUCATIVA
}

\author{
Leda Badilla Cavaría \\ Posgrado en Administración Educativa \\ Universidad de Costa Rica \\ E-mail: lbadilla@cariari.ucr.ac.cr
}

\begin{abstract}
Resumen
Badilla, L. (2006). Fundamentos del paradigma cualitativo en la Investigación Educativa. Revista de Ciencias del Ejercicio y la Salud, 4(1), 42-51. La investigación cualitativa en el campo de la educación es un tema de interés actual, ya que está inmersa en nuestras prácticas profesionales cotidianas. El propósito de este intercambio investigativo permite reconstruir, articular y reflexionar sobre algunos tópicos que fundamentan el enfoque cualitativo de investigación y que están relacionados con nuestros escenarios habituales de trabajo. De manera que primero se realizará una aproximación, desde sus componentes epistemológicos y metodológicos, a la investigación cualitativa, para luego continuar caracterizando este enfoque desde la producción de la información, su análisis, las fuentes de credibilidad de los resultados y la ética que lo sustenta. PALABRAS CLAVES: investigación cualitativa, investigación educativa.
\end{abstract}

Una de las decisiones estratégicas iniciales cuando diseñamos investigaciones se vincula con la elección del enfoque o paradigma que utilizaremos. Como educadores $y$ educadoras estamos en presencia de un enfoque que nos ofrece muchas potencialidades para mejorar nuestra práctica profesional, es el enfoque de investigación cualitativa.

La investigación cualitativa en el campo de la educación es un tema de interés actual, esto porque su práctica se ha popularizado en los últimos años en el ámbito de las ciencias sociales, lo que ha permitido su expansión pero a la vez la profundización en las teorías y las metodologías que la sustentan. Cada día, la investigación cualitativa, responde a más cuestiones de interés educativo-pedagógico y es que la investigación cualitativa está inmersa en nuestras prácticas profesionales cotidianas.

El propósito de este intercambio investigativo permite reconstruir, articular y reflexionar sobre algunos tópicos que fundamentan el enfoque cualitativo de investigación y que están relacionados con nuestros escenarios habituales de trabajo.

Se trata pues de:

- Permitir una aproximación, desde sus componentes epistemológicos y metodológicos, a la investigación cualitativa y de

- Caracterizar este enfoque desde la producción de la información, su análisis, las fuentes de credibilidad de los resultados y la ética que la sustenta.

También haré mención a algunas de las prácticas o estereotipos que con frecuencia se tejen alrededor de las prácticas investigativas de orden cualitativo.

Acercarnos a conocer, reconocer y reflexionar sobre los fundamentos de la investigación cualitativa significa en primera instancia acercarnos a sus posibilidades ontológicas, epistemológicas, metodológicas y pedagógicas. Esto es así porque los problemas y las preguntas de investigación operan en función del enfoque o paradigma 
elegido, en función de nuestro posicionamiento frente a la naturaleza de la realidad, a las formas de interacción entre quien conoce y lo cognoscible, a cómo se conduce la búsqueda del conocimiento y a cómo enseñar y poner en práctica ese conocimiento.

\section{I. ¿Cuáles preguntas iniciales nos hacemos para ubicarnos en el paradigma cualitativo ${ }^{1}$}

\section{Plantearemos la primera de ellas:}

\section{¿Cuál es la naturaleza de la realidad en la que nos posicionamos?}

Esta pregunta es ontológica y se vincula a la naturaleza de la realidad investigada. Para el enfoque cualitativo la realidad social es histórica, relacional, dinámica, variable, local pero articulada a procesos amplios más complejos (económicos, políticos, culturales).

Si nos ocupara el tema de la formación de docentes podríamos preguntarnos ¿Desde cuáles realidades, la comunidad educativa establece los rasgos personales y profesionales de los docentes de la educación básica general?

La segunda pregunta que nos hacemos es la siguiente

\section{¿Qué relación se establece entre quien conoce y lo que es posible conocer para producir teoría?}

Esta pregunta es epistemológica y se vincula directamente con el conocimiento. La reflexión sistemática y fundamentada sobre la

\footnotetext{
${ }^{1}$ Algunos autores (Fernández y Santos 1992. Valles, 1997) señalan que la noción "paradigma" se torna ambigua porque puede incluir una serie de procedimientos, metodologías, éticas, entre otras. De ahí que en este texto la utilizo dejando claro que las posibilidades ontológicas, epistemológicas y metodológicas están en permanente interacción, pero no por ello se convierten en un sistema cerrado, de ahí la conveniencia de utilizar las nociones "perspectivas" o "concepciones filosóficas".
}

práctica aporta explicaciones teóricas, es decir, produce conocimientos bajo las operaciones de construcción, ruptura o constatación. Para lograr lo anterior, se estudian y conocen procesos, se reconoce la pluralidad de valores e intereses que conforman la situación de investigación y que median en los hallazgos producidos, se considera las subjetividades e intersubjetividades.

Siguiendo con el tema de la formación de docentes podríamos afirmar, por ejemplo, que se ha dado un conocimiento dialéctico de la realidad en el momento en que candidatos $\mathrm{y}$ candidatas a docentes han interpretado $\mathrm{y}$ valorado hechos de la realidad para lograr una perspectiva teórica sobre su futuro papel profesional.

El tercer cuestionamiento que nos formulamos es el siguiente

\section{¿Cómo se conduce la búsqueda del conocimiento?}

Esta pregunta es metodológica y como tal se transforma en disciplina que estudia, analiza, construye y reconstruye. En investigación cualitativa no hay una sola metodología. Para ello la investigación se apoya en métodos y técnicas que permitan analizar, explicar e interpretar pensamientos que tengan sentido para las personas que participan en las investigaciones.

Es posible que cuando un grupo de investigadores e investigadoras estudia las expresiones y significados expresados por docentes, que dan sentido a su práctica profesional, estén utilizando una metodología hermenéutica por medio de técnicas como la entrevista y el análisis de contenido.

Otro ejercicio metodológico lo realizamos, por ejemplo, cuando se parte de referentes teóricos sobre el significado de la noción "innovación educativa" y volvemos a dichos referentes, luego de una práctica metodológica que es la que permite reconstruirlos y reinterpretarlos en términos 
teóricos, estaremos en presencia de la metodología de la teoría fundamentada.

La cuarta pregunta que debemos formularnos como investigadoras e investigadores educativos es la siguiente

\section{¿Cuál es la mejor manera de socializar el conocimiento?}

Esta es la pregunta pedagógica. El conocimiento producido se comparte con actores y actoras de la investigación. Buscamos permanentemente aprendizajes.

Entre los planteamientos de la investigación cualitativa consideramos el contexto y su historia, las relaciones e intercambios sociales, las representaciones sociales y el lenguaje, la noción de sujeto inclusivo, el interés investigativo básico y el triángulo ético-émico-investigador o investigadora.

\section{II. ¿Cuáles son los planteamientos básicos de la investigación cualitativa?}

La investigación cualitativa se caracteriza por buscar dimensiones no conocidas o poco conocidas de un hecho social. Estas dimensiones se buscan también a partir de la forma como viven y entienden ese hecho los grupos afectados por él. Entre los planteamientos consideramos el contexto y su historia, las relaciones e intercambios sociales, las representaciones sociales y el lenguaje, la noción de sujeto inclusivo, los intereses básicos y el triángulo ético-émicoinvestigador

\subsection{El contexto y su historia}

La realidad se construye y reconstruye, por ello es necesario conocer los fenómenos que suceden en ella, explicarlos, comprenderlos para poder interpretarlos. El contexto es importante. No interesa llegar a conclusiones genéricas, sino que lo que interesa es conocer a fondo cada situación, cada particularidad.
Los contextos no pueden entenderse sin su historia, de igual manera sucede con los hechos educativos. Las situaciones actuales se pueden comprender si se consideran los actos que sucedieron. Por ello se habla de contextos diacrónicos en el sentido que los hechos cuentan tal y como se ha desarrollado a través del tiempo. Lo contrario serían hechos sincrónicos, donde los fenómenos se describen independientemente del proceso histórico. En el contexto confluyen prácticas lingüísticas, ideológicas, culturales, al mismo tiempo que ocurren hechos externos, como lo son por ejemplo, las significaciones que poseen las personas de la comunidad sobre los y las docentes o bien sobre su función.

\subsection{Las relaciones $e$ intercambios sociales}

Los actos educativos son relacionales. Las situaciones educativas tienen como punto de llegada y de partida una serie de relaciones: interpersonales, de autoridad, de sumisión, de información, de aprendizaje, de enseñanza, de control, de colaboración. Las relaciones se establecen en todos los ámbitos en que operan los actos educativos. Podríamos esperar que las relaciones en los centros educativos sean formales, sin embargo, existen una serie de relaciones informales tan importantes como las formales.

La acción investigativa cualitativa en el campo de la educación, busca situarse en las relaciones cotidianas, ya sea entrando en los espacios comunicativos o reconstruyendo dinámicas interpersonales de las acciones, con esto se crean y recrean las realidades sociales, en nuestro caso como un conjunto de prácticas educativo-pedagógicas situadas, esto es, considerando los procedimientos, métodos, mitos, rituales, que utilizan permanentemente los diferentes actores y actores para construir sus mundos. Las relaciones pueden parecer simples pero por lo general, su complejidad no se observa, dada la diversidad de relaciones que se suceden en las situaciones educativas. Entonces no basta con describir los hechos sino que se hace 
necesario su interpretación y así la posibilidad de transformación.

\subsection{Las representaciones sociales y el lenguaje}

Las representaciones sociales, están constituidos por prácticas e ideas que permiten a las personas orientarse en sus mundos sociales, "aprehender" esos mundos y de este modo establecer los códigos para nombrar y clasificar las cosas u objetos, su historia individual y grupal. Las representaciones sociales son tanto contenidos como procesos que forman parte de las investigaciones cualitativas. Las representaciones intervienen en los actos cognitivos de las personas, ya que despliegan acciones interpretativas, espontáneas $\mathrm{o}$ formales, que son las que permiten formar vínculos sociales concretos. El lenguaje se constituye en el andamio de las interacciones, ya que él posibilita las significaciones y por consiguiente saberes y conocimientos. En otras palabras, el lenguaje posibilita que se sucedan procesos cognitivos, vocabularios narrativos y contextos de aplicación.

La investigación cualitativa no utiliza ni códigos ni lenguajes elaborados fuera del universo de interacciones de sus actores y actoras.

\subsection{La noción de "sujeto" inclusivo}

Los sujetos sociales, hombres y mujeres, son quienes configuran los mundos sociales por medio del significado que le dan a sus acciones, a las cosas que les rodean, a las otras personas. En nuestro caso, ese mundo educativo, se basa por lo general, en negociaciones conscientes e inconscientes, entre actores y actoras, para buscar acuerdos sobre los significados de ese mundo. Esta condición no es fija, se reconstruye de manera intersubjetiva y relacional. Actores y actoras educativos se vinculan por relaciones que unen $y$ articulan procesos cognitivos, actitudinales, de valores entre otros, que surgen al interior de las situaciones educativas. Esta relación es inclusiva ${ }^{2}$, se constituye a partir de un nosotros "y" ellos, ya que los sujetos educativos son sociales a la vez que sujetos políticos, psíquicos, culturales, entre otros.

El sujeto no se separa del objeto, ya que quien conoce no se separa de lo que conoce. Existe un principio de inclusión, por ejemplo, de quien observa en lo observado.

\subsection{Los intereses: describir, comprender, explicar, interpretar}

Los procesos sucesivos para producir datos y configurar textos significativos, se vinculan a acciones como describir, comprender, explicar e interpretar. La investigación cualitativa se interesa por la descripción como proceso para elaborar datos. Es posible que las descripciones solicitadas por investigadores sobre la figura de docentes, dirija a éstos a releer una y otra vez los textos producidos, para hacer comprensibles las respuestas brindadas y de este modo explicar algunos de los rasgos presentes en "la figura del docente" que a la vez llegan a interpretar como "una fragmentación de los rasgos que denota que no existe una percepción muy clara de la profesión por parte de padres, madres $y$ estudiantes". Para llegar a esta interpretación ha sido necesario identificar textos significativos, que de algún modo se articulan en un contexto (investigativo, discursivo, institucional $\mathrm{u}$ otros) donde se manifiestan ciertas reglas implícitas, explícitas o simbólicas.

En la investigación cualitativa el proceso interpretativo se va sucediendo casi con la producción de los datos.

\subsection{El triángulo: lo ético, lo émico y quien investiga}

\footnotetext{
${ }^{2}$ Noción que trabaja de Alba (2000) en su artículo “ Sujeto político, sujeto educativo: claves a partir de dos conjunciones"
} 
Los conceptos "ético" y "émico" se utilizan con alguna frecuencia en investigación cualitativa para representar dos tipos de relaciones. La relación ética, alude a lo externo, a aquello que quien investiga aporta desde el exterior, esto es, a los temas de la disciplina, de autores o de otros desde una perspectiva descriptiva y general. Podríamos mencionar las tendencias internacionales para la enseñanza de la matemática, el currículo oficial para la educación ambiental, la didáctica como campo disciplinar. Estas referencias requieren de contextualización, por lo que se dirigen a la perspectiva émica, esto es, a los temas propios de actores y actoras de la situación educativa por investigar. Los significados surgen desde dentro, en nuestro caso, desde la cultura escolar propia, desde los actores del currículo vinculado a la educación ambiental, desde los y las egresadas de las escuelas normales, desde los docentes que enseñan matemática, desde quiénes aprenden las ciencias naturales.

Quien investiga, realiza articulaciones de ético a lo émico y viceversa, con un propósito contextualizador y de reformulación permanente de los referentes teóricos.

\section{La negociación}

En las investigaciones cualitativas el conocimiento de la realidad se construye y reconstruye por medio de las interacciones que se suceden entre diferentes actores y actoras. La negociación se refiere a consensos llevados a cabo antes, durante y después del trabajo de campo para establecer ciertas condiciones y principios éticos en los que se desarrolla la producción de los datos, su análisis, discusión y socialización de los mismos. Se procura que todas las personas participantes en la investigación, acepten la investigación como un acto que produce conocimientos y por consiguiente tienen un potencial para mejorar las prácticas educativas o sociales. Se garantiza que la información que pueda suponer algún riesgo será confidencial.

Este proceso de negociación asegura en gran parte la producción de la información y la colaboración de quienes participan. Las características deseables de la negociación refieren a que sea participativa, explícita y flexible (Fernández y Santos, 1992).

El acceso al campo también requiere de otras negociaciones, aunque los problemas de investigación pueden estudiarse en diversos contextos particulares, buscamos algunos criterios para elegir el campo donde se desarrollará la investigación, que no depende solo de nosotros sino de otros factores como lo son el acceso a instituciones y a fuentes personales y documentales entre otras.

\section{El diseño cualitativo de investigación}

Los referentes anteriores forman parte del diseño investigativo, sin embargo, esta parte es más operativa, de ahí que aparezca como una sección separada. En el diseño consideraremos la definición del problema por investigar y los procedimientos para producir la información, analizarla y asegurar su credibilidad además de las características del informe final.

\subsection{El problema}

En las investigaciones, cualquiera sea su naturaleza, se hace necesario definir el problema por investigar. En el caso de la investigación educativa cualitativa al problema se le asigna cierta provisionalidad, porque es la producción de la información y su respectivo análisis lo que permite acercarse a él y orientarse. Sus fronteras no se delimitan. A veces se habla de problemáticas, no de problemas específicos.

La selección del problema significa enfocar la atención en fenómenos o situaciones educativas específicas vinculadas de algún modo con referentes conceptuales. Por lo general el problema y los subproblemas se plantean en forma interrogativa. 
El contenido de las preguntas guía la atención y el pensamiento de manera más o menos concreta, ya que en el desarrollo de la investigación pueden surgir otras interrogantes, que si bien no desplazan el problema o cuestionamiento central que motiva la investigación, sí se pueden considerar como subproblemas $\mathrm{o}$ interrogantes específicas de la investigación.

Algunos autores como Stake, (1998), denominan a éstas como "preguntas temáticas" ya que dirigen la atención a los problemas. El autor aclara que los problemas son más concretos y los temas más abstractos.

Cuando se decide utilizar hipótesis, éstas son de trabajo (no formales) y los referentes teóricos que las acompañan tiene un valor instrumental.

\subsection{Los acercamientos teóricos}

Toda investigación requiere de constructos o referentes conceptuales que guíen las etapas siguientes de la investigación. En la investigación de naturaleza cualitativa los acercamientos teóricos son inicialmente temporales, ya que lo que comúnmente se conoce como marco o premisas teóricas son susceptibles de ser modificadas conforme se avanza en los hallazgos. Esto significa que la construcción de la teoría, además de que procede de los datos, es elaborada sistemáticamente en relación con ellos $^{3}$ a lo largo del proceso investigativo. Podría señalarse que la elaboración de la teoría también es un proceso investigativo (Ruiz, 1996).

\subsection{La selección de los y las participantes}

En el tipo de investigación que nos ocupa se requiere que quien investiga determine las características relevantes que poseen grupos, actores y actoras de la investigación. Esto

\footnotetext{
${ }^{3}$ Esta sistematización la plantea la teoría fundamentada o "Grounded Theory"
}

permite ubicarse en aquellas situaciones y personas que produzcan información relevante para dar respuesta a las preguntasproblema planteadas y por consiguiente a la teoría que se busca.

Los criterios de selección se constituyen en criterios de comprensión en el tanto son pertinentes y no representativos en términos estadísticos. Por pertinencia entendemos que los textos o discursos producidos por las y los actores muestren relaciones importantes, simbolismos, representaciones, es decir, información suficiente y pertinente.

El procedimiento señalado se denomina selección teórica. Las y los investigadores buscan grupos, personas o sucesos específicos, con ciertas características, conocen dónde y cuándo encontrarlos, pero lo más importante es que se produzca la información más relevante para lo que se persigue. Esta selección teórica no acaba hasta que deja de aparecer nueva información, es decir hasta que se alcanza un nivel de saturación y una riqueza de información. La selección no obedece a normas fijas, acepta que el número de participantes puede variarse si se requiere mejorar la riqueza de la información o bien puede disminuirse si se ha alcanzado la saturación teórica ${ }^{4}$.

\subsection{Las técnicas a utilizar}

Las técnicas identificadas con la investigación cualitativa son la observación y la entrevista a profundidad. Esto nos sugiere que las preguntas que se le hacen a la realidad son abiertas. A estas preguntas las guían teorías de la acción social que están orientadas por experiencias compartidas por diferentes participantes, por referencias del acontecer educativo o por valoraciones que se le atribuyen a ese acontecer.

\footnotetext{
${ }^{4}$ La información se analiza casi al mismo tiempo que se produce, de ahí que pronto se evidencia su repetición.
} 
Otros métodos o técnicas ${ }^{5}$ que se utilizan con frecuencia son:

- las historias de vida

- el análisis de contenido

- los grupos focales

- el análisis semiótico del discurso

- el estudio de casos

- la investigación-acción

- los métodos no intrusivos

\subsection{El trabajo de Campo}

Por trabajo de campo entendemos el período de tiempo y el modo dedicado a producir y registrar datos. Se considera como una situación metodológica y un proceso. No todos los acontecimientos que se suceden pueden ser controlados por el o la investigadora.

Se trata de la permanencia continuada y prolongada por parte de quien (es) investiga (n) entre el grupo humano de participantes.

En el enfoque cualitativo el trabajo de campo y la elaboración técnica no se preparan.

El trabajo de campo implica un proceso de socialización, ya que las y los investigadores desarrollan una serie de aprendizajes del contexto cultural o situación investigativa. Se aprenden: ciertas normas, códigos, sistemas de comportamiento, comunicaciones verbales y no verbales. El acceso al campo nos inicia con una serie de significaciones que poco a poco vamos desconstruyendo.

\section{6. El análisis e interpretación de la información producida}

\footnotetext{
5 Por la rigurosidad investigativa deseable, diferenciamos las nociones de metodología, método y técnica, ya que con frecuencia se utilizan indistintamente. La metodología tiene una referencia epistemológica, se hace posible por el método que brinda coherencia a los procedimientos y se operativiza por las técnicas.
}

Esta tarea es compleja y demanda bastante tiempo y paciencia. Con frecuencia la cantidad de información nos agobia y no sabemos cómo iniciar la lectura-relectura y la desconstrucción de la información, que es la que nos permite reconocer claves de interpretación. Al mismo tiempo observamos que la información es heterogénea, porque cada quien posee sus visiones propias de la realidad que nos ocupa.

La desconstrucción inicia con una dialéctica entre la información (los datos) y su comprensión-traducción-interpretación. Al respecto se pueden señalar dos acciones. Una se vincula con descubrir significantes de uno o varios fenómenos (situaciones educativas) y la otra a identificar categorías teóricas relevantes o dimensiones que emergen del análisis sistemático de la información y que a la vez nos ayudan a determinar algunos constructos teóricos que nos hacen volver a los acercamientos teóricos iniciales para reconstruirlos. Al inicio del análisis se suelen identificar muchas categorías, sin embargo conforme se sigue con la tarea desconstructora algunas categorías se asimilan a otras, dada su evidente articulación conceptual o práctica.

Lo anterior exige de investigadores e investigadoras una actitud de apertura a significantes no previstos que con frecuencia nos asombran.

Conviene recordar que la información no es la realidad misma que se desea investigar, sino que son distintos pareceres $o$ significantes acerca de ella. La interpretación nos permite conocerla desde las distintas perspectivas que viven las y los actores de la investigación.

\subsection{La credibilidad de la información}

Investigadoras e investigadores cualitativos reconocen la necesidad de que la información que se produce sea creíble, para ellos se preocupan de la coherencia y rigurosidad en la interpretación, lo que significa que las consecuencias de utilizar los 
resultados deben ser consideradas parte de la responsabilidad de quien guía la investigación. Lo anterior exige mucho rigor en el camino investigativo, por ellos es que desarrollamos ciertas "estrategias" y ciertos "procedimientos" que nos garantizan la credibilidad de las interpretaciones a las que llegamos.

Algunos autores y autoras centran la credibilidad de la interpretación de la información en la coherencia entre la ontología, epistemología y metodología que rige la investigación; otros la dirigen a la rigurosidad con la que la información se produce y analiza y otros tienden a centrarse en la o el investigador como instrumento central tanto en la producción de información como en el proceso de análisis. La credibilidad de los hallazgos se dirige a la utilización de técnicas seguras; a las calidades y experiencia de las y los investigadores y a la perspectiva paradigmática en que orienta la investigación (Patton, 1990).

Para argumentar la credibilidad ${ }^{6}$ de las descripciones e interpretaciones se utiliza la noción triangulación, que consiste en una serie de estrategias por medio de las cuales las y los investigadores compensan el sesgo que podría permear la información. Por ejemplo la utilización de varias técnicas para estudiar un mismo fenómeno, permite comprensiones e interpretaciones más ricas de las situaciones investigadas. Recuérdese que quien investiga es quien construye significados. La estrategia de la triangulación se operativiza por una serie de procedimientos como los siguientes (Denzin y Guba, 1994. Patton, 1990. Stake, 1998) :

Triangulación de las fuentes de datos, se observa si la situación de investigación sigue

\footnotetext{
6 Credibilidad y autenticidad se corresponden con validez interna y externa del paradigma cuantitativo: La credibilidad se asocia con la validez interna ya que considera "las verdades" de la investigación. La transferibilidad con la validez externa ya que mira la aplicabilidad de los resultados. La dependencia como confiabilidad porque se vincula con la consistencia de los datos y la conformidad, como objetividad ya que se refiere al problema de la neutralidad.
}

siendo la misma en otros espacios, otros momentos o con otras personas. Responde a preguntas como por ejemplo ¿Qué cambios hacen que las y los docentes pongan en práctica métodos inductivos para la enseñanza de las ciencias? En este caso la triangulación de las fuentes de datos nos permite observar si el significado es el mismo cuando lo encontramos en otras circunstancias. Se recurre a datos diferenciados por haber sido producidos y analizados en diferentes tiempos, espacios y por diferentes investigadores.

Triangulación de teorías, se da cuando se recurre a personas, comisiones especializadas $\mathrm{u}$ otras que poseen puntos de vista bajo teorías alternativas. Cada quien realiza diferentes interpretaciones sobre la misma situación, de modo que se produce alguna triangulación de la teoría. Por ejemplo, la información producida por el $\mathrm{y}$ las investigadoras sobre el docente como investigador de su práctica desde la investigación acción, puede ser triangulada por pedagogos críticos y por pedagogos constructivistas. Ambos grupos de especialistas han encontrado algunos significados similares, lo que indica que se ha dado un proceso de triangulación, aunque hayan sido pocos los significados comunes.

Triangulación de técnicas, se sucede cuando se aumenta la confianza en la interpretación y esto se facilita con múltiples técnicas que se contrastan y que se utilizan para abordar una misma situación. Por lo general, la información producida por las diferentes técnicas respaldan en parte las descripciones e interpretaciones. Por ejemplo, la información producida por medio de las guías de observación utilizadas en el desarrollo de la enseñanza de las ciencias naturales en la educación básica se contrastan con la información producto de las entrevistas realizadas a docentes formadores.

Triangulación por investigadores, se pone en práctica cuando le presentamos a algún o 
alguna investigadora los resultados de nuestras interpretaciones $\mathrm{u}$ observaciones para su análisis.

Lo más importante de la triangulación es que nos exige una y otra vez revisar la información producida. Los procedimientos de triangulación muchas veces han servido para más interpretaciones adicionales antes que corroborar significados encontrados.

\subsection{El informe final}

No existe una forma única de redactar el informe de investigación cualitativa, sin embargo, como en cualquier informe de investigación deben quedar desarrolladas las fases de

- posicionamiento onto-epistemológico y metodológico

- diseño: problema, preguntas de investigación, acercamientos teóricos

- trabajo de campo: producción de la información, triangulación

- análisis: preliminar, relectura, emergencia de categorías

- conclusiones para el contexto

- devolución de la información a participantes

Es importante que la redacción y organización del informe ayude a lectores y lectoras a comprender la investigación. El informe es competencia y responsabilidad del equipo investigador, aunque en la investigación cualitativa es deseable la devolución de los resultados para su discusión, a los y las participantes, no siempre se cumple con esta práctica.

Las preguntas de la investigación nos han servido para estudiar la complejidad de la situación educativa. La diacronía de los hechos nos ayuda a desarrollar el informe, el diario de campo se constituye en un apoyo decisivo.

Por lo general hacemos un esquema escrito de las secciones que contendrá el documento final, sin embargo, este esquema no es rígido y a veces varía conforme avanzamos en el análisis de la información producida. Las categorías emergentes podrían constituirse en capítulos del informe.

\section{El diario de campo}

Se constituye en un instrumento de registro fundamental en este tipo de investigación. En él se registran hechos que suceden en forma diacrónica, las ideas inmediatas que se nos ocurren, aspectos programáticos de la investigación, comentarios propios o de otras personas, observaciones de acontecimientos, comentarios informales, temores, confusiones, soluciones, afectos, entre otros. Las y los etnógrafos siguen algunas reglas para el registro, por ejemplo, en el diario se "registra a diario", esto es, la información se guarda el menor tiempo posible en nuestra memoria. También se pone mucha atención a la identidad de quién ha dicho o hecho algo de interés.

Con respecto a la estructura del diario Mucchielli (1996) sugiere cuatro apartados: la descripción de contextos, las notas personales, las notas metodológicas y las notas teóricas.

\section{La Etica}

La práctica investigativa exige identificar ciertos valores y principios de acción que la orientan. Los valores son los de respeto y buen trato a participantes, los consensos, la valoración de las ventajas y riesgos de participar, la confidencialidad de la información. Pero además, se señalan tres tipos de relaciones que se dan en las investigaciones cualitativas ellas son (Mucchielli, 1996):

- la relación entre quien investiga y su investigación, vinculada a los intereses que mueven la investigación

- la relación entre quien investiga y los actores y actoras de la investigación, se asume como un compromisos al ocupar 
un espacio en la situación investigativa. En ella se consideran intereses políticos, privilegios que puedan suscitarse, la presencia ante situaciones comprometedoras que pueden suscitar que investigadores se conviertan en cómplices, testigos o informantes.

- la relación entre quien investiga y la producción de los datos, se aborda desde la redacción de informes, la toma de decisiones técnicas, ideológicas y éticas, esto es qué y cómo se va a decir.

Además es indispensable comunicar la condición emergente de la investigación cualitativa, que hace que los encuentros no se reduzcan a unos pocos sino que se realizan tantos como los necesite el diseño. Otros de los aspectos que se enuncian y se discuten con los participantes se refieren a los fines y propósitos de la investigación, la concreción de la metodología en la práctica, la emergencia de tiempos y espacios, la difusión social de la información producida, la propiedad intelectual de los hallazgos.

\section{REFERENCIAS}

Alonso, L.E. (1998) La mirada cualitativa en sociología. Madrid: Fundamentos

de Alba, A (2000) Sujeto político, sujeto educativo: claves a partir de dos conjunciones. En : de Alba, A. (coord.) El Fantasma de la Teoría. Cuadernos de construcción conceptual en educación. México: Plaza y Valdes Editores

Denzin, N. y Guba N. (Ed) (1994) Handbook of Qualitative Research. Thousands Oaks: Sage

Fernández, J. y Santos, M. (1992) Evaluación cualitativa de programas de educación para la salud. Málaga: Ediciones Aljibe

Mucchielli, A. (1996) Diccionario de Métodos Cualitativos en Ciencias Humanas y Sociales. Madrid: Editorial Síntesis

Patton, M. (1990) Qualitative Evaluation and Research Methods. Newbury Park: Sage Publications

Ruiz, J.I. (1996) Metodología de la Investigación Cualitativa. Bilbao: Universidad de Deusto.

Stake, R.E. (1998) Investigación con estudio de casos. Madrid: Ediciones Morata

Valles, M. (1997) Técnicas cualitativas de investigación social. Reflexión metodológica y práctica profesional. Madrid: Editorial Síntesis

Velasco, H. Díaz de Rada, A. (1997) La lógica de la investigación etnográfica. Un modelo de trabajo para etnógrafos de la escuela. Madrid: Editorial Trotta. 